\title{
Maternal outcome in thyroid dysfunction in admitted antenatal women in tertiary care centre: an observational study
}

\author{
Juhi Agarwal, Deepika Kesaraju, Poorva Badkur*
}

Department of Obstetrics and Gynecology, Gandhi Medical College, Bhopal, Madhya Pradesh, India

Received: 10 June 2020

Accepted: 08 July 2020

\section{*Correspondence:}

Dr. Poorva Badkur,

E-mail: poorvadoc@gmail.com

Copyright: (C) the author(s), publisher and licensee Medip Academy. This is an open-access article distributed under the terms of the Creative Commons Attribution Non-Commercial License, which permits unrestricted non-commercial use, distribution, and reproduction in any medium, provided the original work is properly cited.

\begin{abstract}
Background: Thyroid disorders constitute one of the most common endocrine disorders in pregnancy. Aim of this study was to identify thyroid dysfunction as a maternal health problem. Objective of this study was to study the maternal outcomes in these patients.

Methods: The main source of data are the patients admitted in Sultania Zanana Hospital, GMC Bhopal. The study was an observational prospective study conducted from March 2018 to February 2019.

Results: The present study was conducted in department of obstetrics and gynecology, Gandhi Medical College and associated Sultania Zanana Hospital Bhopal for the period of 1 year. The study could enroll 400 females fulfilling the inclusion criteria during the study period.

Conclusions: In developing countries like India where there is prominent micronutrient deficiency, iodine deficiency further aggravates the condition and carries a great potential to show deleterious impacts on maternal and fetal outcomes, thus serving a major health problem. Considering the substantial increase in short term as well as long term perinatal morbidity, a felt need for creating awareness not only on adverse outcomes but also detection of the disorder in first trimester is required. The desired perinatal outcome can be achieved by early detection and prompt treatment of the disorder. Henceforth, universal screening of all antenatal women in the first trimester rather than targeted case finding should be recommended at all the health care facilities.
\end{abstract}

Keywords: Antenatal, Hypothyroidism, Maternal, Outcomes, Thyroid dysfunction

\section{INTRODUCTION}

Thyroid disorders constitute one of the most common endocrine disorders in pregnancy. During pregnancy, the maternal thyroid gland faces several metabolic, hemodynamic and immunologic changes. Furthermore, in women who suffered from thyroid dysfunction prior to pregnancy, the hormonal changes are magnified. There is an intimate relationship between the maternal and fetal thyroid gland. Thus, abnormal maternal thyroid hormone can influence the outcome for mother and fetus at all stages of pregnancy. ${ }^{1}$ Women with both overt and subclinical hypothyroidism are at increased risk of pregnancy related complication such as abortions, preeclampsia, preterm labor, placental abruption and PPH. Thyroid hormone results in fetal and maternal complication. Deficiency of which can result in irreversible brain damage with mental retardation and neurological abnormalities. ${ }^{2}$

Hyperthyroidism in pregnancy is less common than hypothyroidism and can lead to complications like congestive heart failure, thyroid storm, hyperemesis gravidarum, preeclampsia, preterm delivery, fetal growth restriction, still birth, fetal and neonatal thyrotoxicosis. Thyroid dysfunction often goes unseen in pregnant women partially due to the nonspecific signs and symptoms and also due to the hypermetabolic state of 
pregnancy. Thus, making thyroid function test essential to know the thyroid status in pregnancy and also to detect the subclinical disease.

\section{Aims}

The aim of this study was to identify thyroid dysfunction as a maternal health problem.

\section{Objective}

The objective of this study was to study the maternal outcomes in these patients.

\section{METHODS}

The main source of data are the patients admitted in Sultania Zanana Hospital, GMC Bhopal. The study was an observational prospective study conducted from March 2018 to February 2019.

\section{Inclusion criteria}

- All the consenting pregnant women admitted in the department of obstetrics and gynecology at Sultania Zanana hospital.

- Known case of thyroid disorders who are on treatment.

\section{Exclusion criteria}

- Known cases of other endocrinological disorders

- Gestational trophoblastic diseases

- Multiple pregnancy

- Chronic hypertension.

\section{Procedure}

After obtaining an informed consent, 400 admitted antenatal patients were randomly selected for the study

A detailed history of signs and symptoms, obstetric history, Menstrual history, past and personal history, family history was taken

- Physical examination was done

- Serum thyroid profile testing was done

- First trimester TSH: 0.1-2.5 mIU/L second trimester TSH: 0.2-3.0 $\mathrm{mIU} / \mathrm{L}$ third trimester TSH: $0.3-3.0$ $\mathrm{mIU} / \mathrm{L}$

- Based on the Trimester specific TSH, total T3, T4 patients were grouped into subclinical hypothyroidism, overt hypothyroidism, subclinical hyperthyroidism, overt hyperthyroidism

- Patients with thyroid disorders were started on treatment and were further followed up for maternal and fetal outcome.
The following maternal outcome variables were observed

- Abortion

- Pregnancy induced hypertension

- Antepartum hemorrhage

- Preterm labor pains

- Oligohydramnios.

\section{RESULTS}

The present study entitled "maternofoetal outcome in thyroid dysfunction in antenatal women- an observational study" was conducted in department of obstetrics and gynecology, Gandhi Medical College and associated Sultania Zanana Hospital Bhopal for the period of 01 year. The study could enrol 400 females fulfilling the inclusion criteria during the study period.

\section{Distribution of patients according to age}

Majority of patients in present study belonged to age group of 21 to 25 years $(68.5 \%)$ followed by $14.8 \%$ patients in less than 20 years of age group. Only $4 \%$ patients belonged to more than 30 years of age (Figure 1).

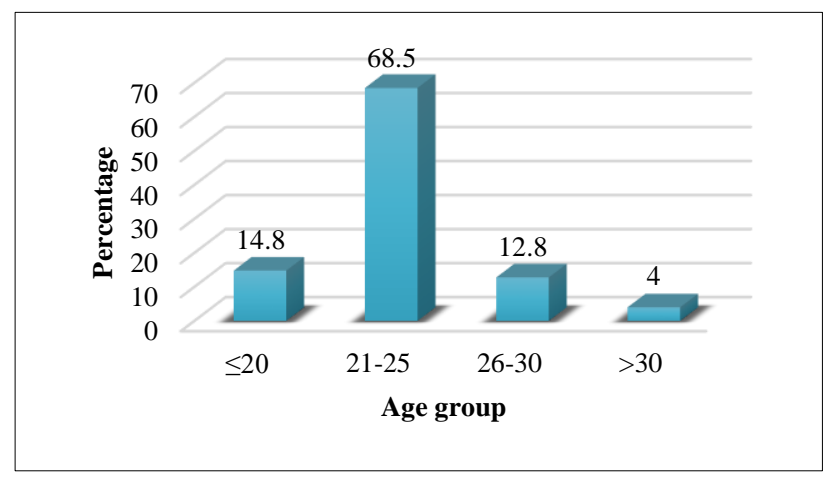

Figure 1: Distribution of patients according to age.

\section{Distribution according to obstetric history}

Maximum i.e. $45.8 \%$ females were primigravida. Only $39.5 \%$ and $14.8 \%$ females were second and third gravida respectively (Figure 2).

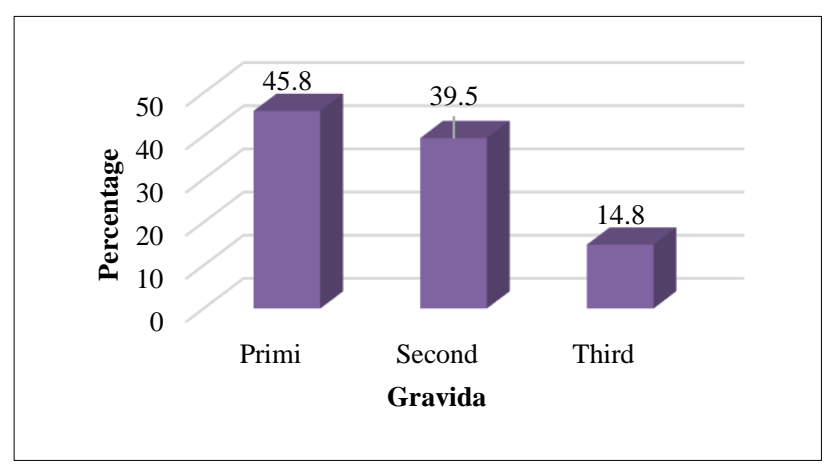

Figure 2: Distribution according to obstetric history. 


\section{Distribution according to trimester of presentation}

In present study, $86.5 \%$ patients presented in third trimester whereas $8.3 \%$ and $5.3 \%$ patients presented in second trimester respectively (Figure 3).

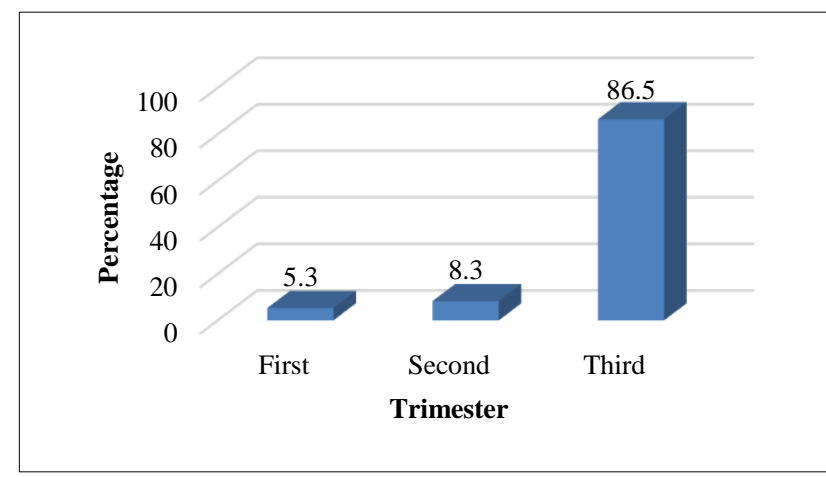

Figure 3: Distribution according to trimester of presentation.

\section{Distribution according to proportions of thyroid disorders}

Out of 400 patients, thyroid disorders were observed in $57(14.4 \%)$. Subclinical hypothyroidism was the most common disorders observed in $9.8 \%$ patients followed by overt hypothyroidism and overt hyperthyroidism in $4.3 \%$ and $0.3 \%$ patients respectively (Figure 4 ).



Figure 4: Distribution according to proportions of thyroid disorders.

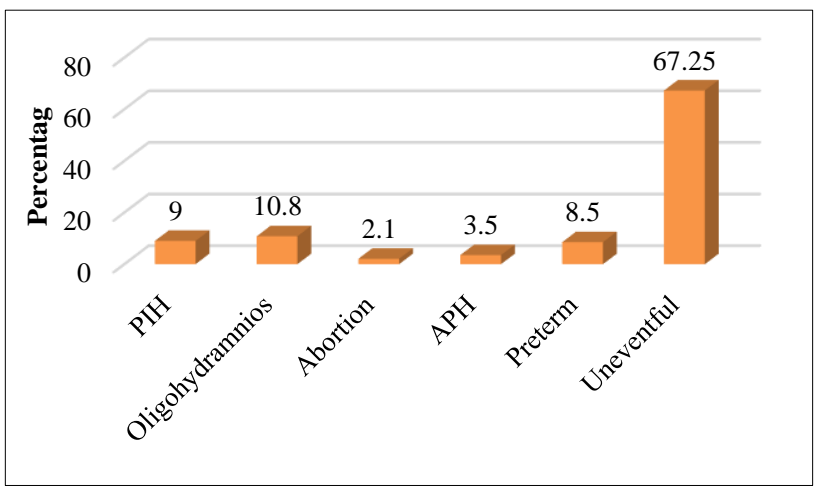

Figure 5: Distribution according to maternal outcome.

\section{Distribution according to maternal outcome}

In present study, outcome was uneventful in $67.25 \%$ patients regardless of thyroid status, and the most common adverse maternal outcome was oligohydramnios (10.8\%) followed by PIH and preterm in $9 \%$ and $8.5 \%$ cases respectively (Figure 5).

\section{Association of gestational age with thyroid disorder}

Out of 39 cases with subclinical and overt hypothyroidism, $87.2 \%$ and $82.4 \%$ patients respectively presented in third trimester. In the present study maximum patients reported to hospital in third trimester $(\mathrm{p}>0.05)$ (Figure 6).

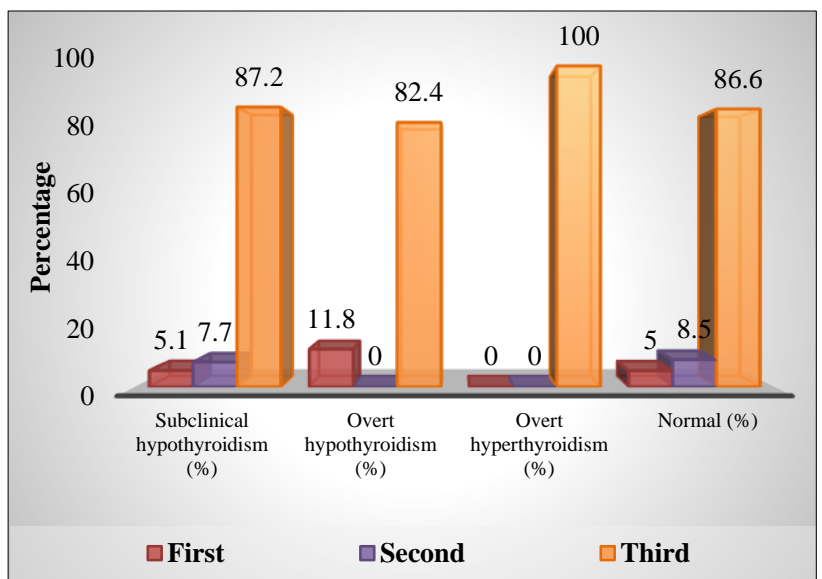

Figure 6: Association of gestational age with thyroid disorders.

Trimester-wise maternal outcome amongst subclinical hypothyroid and euthyroid

The present study observed significantly higher incidence of abortions and preterm births in subclinical hypothyroid females as compared to euthyroid females during first trimester $(\mathrm{p}<0.05)$ (Figure 7).

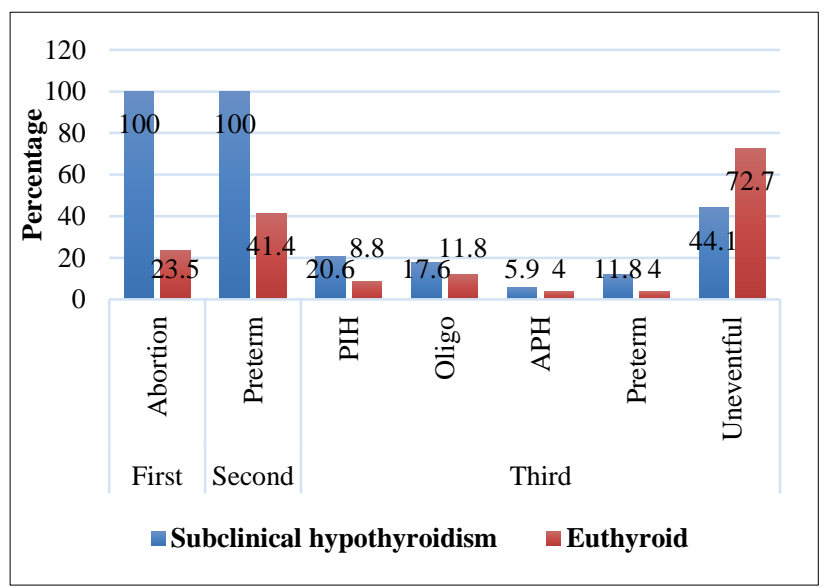

Figure 7: Trimester-wise maternal outcome amongst subclinical hypothyroid and euthyroid. 


\section{Trimester-wise maternal outcome amongst overt hypothyroid and euthyroid females}

The present study observed significantly higher incidence of abortions in overt hypothyroid females as compared to euthyroid females $(\mathrm{p}=0.05)$ (Figure 8).

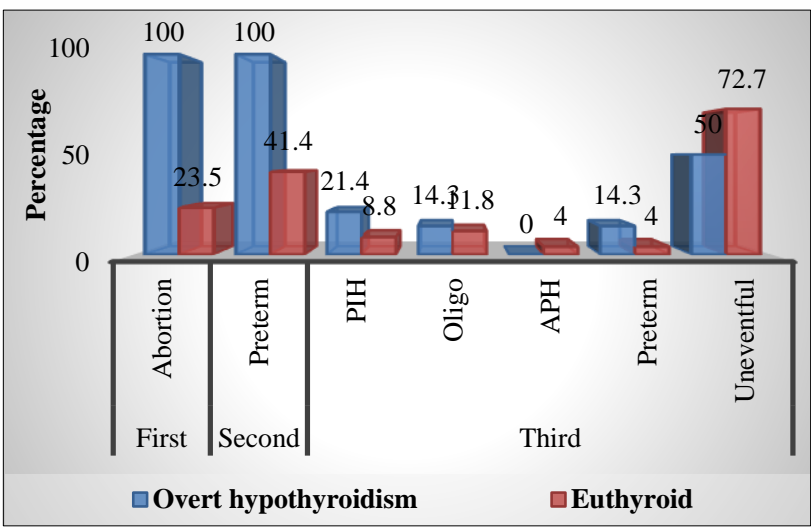

Figure 8: Trimester-wise maternal outcome amongst overt hypothyroid and euthyroid females.

\section{DISCUSSION}

The present study entitled "maternofetal outcome in thyroid dysfunction in admitted antenatal women - an observational study" was conducted in department of obstetrics and gynecology, Sultania Zanana Hospital, Gandhi Medical College Bhopal. 400 patients admitted in the study centre and fulfilling the inclusion criteria over the duration of study period were enrolled in the study. The maternal and fetal outcome of females with thyroid disorders were compared with euthyroid females. Thyroid disorders are the second common cause of endocrine dysfunction in pregnancy after diabetes mellitus. ${ }^{1}$ Thyroid disorders in mother pregnancy may lead to adverse pregnancy outcome and can affect fetal development.

\section{Thyroid disorders}

The present study observed thyroid disorders in 57 Patients (14.4\%). Subclinical hypothyroidism was the most common disorders observed in $9.8 \%$ patients followed by overt hypothyroidism $(4.3 \%)$. Only 1 case of hyperthyroidism was enrolled in present study. Sreelatha $\mathrm{S}$ et al included all cases of thyroid disorders; of the 100 cases, 96 cases were hypothyroid and only 4 cases were hyperthyroid. ${ }^{2}$ In a study conducted by Rajalakshmi MS et al out of 300 selected women overt hypothyroid patients were $14(4.7 \%)$ and subclinical hypothyroid patients were $286(95.3 \%)$ 68. 59 The prevalence of hypothyroidism in current study was high i.e. $14.1 \%$ which is consistent with other studies mentioned above. It can also be concluded from above that the prevalence of hypothyroidism is more so in developing countries like India as compared to developed countries. Further supporting this is an epidemiological study of 11 cities from 9 states in India conducted by Dhanwal et al which concluded that there is high prevalence of hypothyroidism (13.13\%) in India, majority being subclinical in pregnant women during $1^{\text {st }}$ trimester. ${ }^{3}$

The prevalence of hypothyroidism in current study was high i.e., $14.1 \%$ which is consistent with other studies mentioned above. It can also be concluded from above that the prevalence of hypothyroidism is more so in developing countries like India as compared to developed countries. Further supporting this is an epidemiological study of 11 cities from 9 states in India conducted by Dhanwal et al, which concluded that there is high prevalence of hypothyroidism (13.13\%) in India, majority being subclinical in pregnant women during 1 st trimester. $^{3}$

\section{Maternal outcome}

In present study, $54.4 \%$ and $29.2 \%$ cases of thyroid disorders and without thyroid disorders were associated with complications.

In present study, authors observed no statistical association of thyroid disorders with obstetric history and gestational age $(p>0.05)$. However, $86.5 \%$ of the patients in my study reported in third trimester and had undergone thyroid screening for the first time in third trimester. Amongst which $6.5 \%$ of patients had reported with hypothyroidism when the complications had already set in, implying an early detection and prompt treatment could possibly have averted the adverse outcomes. The present study observed significantly higher incidence of abortions and preterm births in subclinical hypothyroid females as compared to euthyroid females during first trimester $(p=0.05)$ but the incidence of abortions was higher in overt hypothyroid patients 63 as compared to euthyroid cases $(\mathrm{p}<0.05)$.

This high incidence was because all patients of first trimester reported for the first time and were not on treatment and all patients of subclinical hypothyroidism were not on treatment. The study enrolled only one case of hyperthyroidism undergoing treatment and the outcome was uneventful in respective case.

\footnotetext{
Abortion

Due to the smaller number of pregnant women reporting in the early gestation age, trimester specific randomization of admitted pregnant women was not done in the current study, Yet The present study observed significantly higher incidence of abortions in both subclinical and overt hypothyroid patients. Tanuja PM et al, reported $1.7 \%$ abortions amongst hypothyroid females. ${ }^{15}$ Sreelatha $\mathrm{S}$ et al in their study reported abortion in $2.1 \%$ cases. ${ }^{1}$ This could be attributed to presence of antibodies to thyroid peroxidase (TPO-AB) or thyroglobulin.
} 


\section{Pregnancy induced hypertension}

The present study observed significantly higher incidence of PIH during third trimester amongst patients with subclinical hypothyroidism but not in over hypothyroid cases. Since the cases with overt hypothyroidism were treated as soon as diagnosed, no significant increase in adverse maternal outcome was observed inthem. Present study reported PIH in $20.6 \%$ cases of subclinical hypothyroidism. Similar scenario has been observed in other studies like Wilson et al, reported that there was a significant association between subclinical hypothyroidism and severe preeclampsia (p value $=0.03) .{ }^{16,17}$ Meena DS et al, in their study observed that $30 \%$ of cases had PIH. ${ }^{6}$ Sreelatha $\mathrm{S}$ et al and Ozdemir $\mathrm{H}$ et al reported $14.7 \%$ and $14.5 \% \mathrm{PIH}$ in subclinical hypothyroid patients respectively. ${ }^{2}$ Rajalakshmi MS et al, also reported $5.5 \%$ cases of preeclampsia in hypothyroid patients. ${ }^{18}$

PIH in pregnancy associated with thyroid disorders could be explained by potentiating beta adrenergic role of thyroid hormones. In hypothyroid state, the density of alpha-1 adrenoceptors increases whereas beta adrenoceptors are decreased on vascular beds. Action of alpha adrenoceptors mainly involves smooth muscle cell contraction, causing vasoconstriction in the blood vessel. ${ }^{2}$

\section{Antepartum hemorrhage}

The present study observed significantly increased risk of APH amongst patients with subclinical hypothyroidism. This could be due to defective placentation which causes increased susceptibility of utero placental interface to both thrombosis and hemorrhage. Although the pathogenesis of antepartum hemorrhage is multifactorial, In patients with thyroid dysfunction, several factors may exaggerate such pathogenesis which include. Tissue factor production in response to aberrant vascular endothelial growth factor and inflammatory cytokine release that promotes thrombosis shallow extra villous trophoblasts invasion (EVT) may lead to placental ischemia and haemorrhage leading to thrombin production locally, which mediates the degradation of extracellular matrix, thus triggering pre-mature placental separation from the uterus.

\section{Preterm labor}

The present study observed significant risk of preterm labor in subclinical hypothyroidism ( $\mathrm{p}$ value=0.04) but not in overt hypothyroidism may be because these patients were under treatment since first trimester.

Saki F et al, evaluated the prevalence of thyroid diseases in pregnancy and its outcomes in south of Iran and observed that clinical hypothyroidism was associated with significant risk of preterm delivery $(\mathrm{p}=0.045) .{ }^{19}$ Sreelatha $S$ reported $3.1 \%$ cases of preterm labor amongst subclinical hypothyroidism. ${ }^{20}$
Although the above discussed complications are known to have multifactorial causes and despite the certain limitations of the study, it can still be concluded from the data of present and various other studies that thyroid disorders contribute major role in aggravating the disease and thus treatment can bring down the adverse maternal and fetal outcomes immensely.

\section{CONCLUSION}

Thyroid disorders in pregnancy, especially hypothyroidism have high prevalence in world population. In developing countries like India where there is prominent micronutrient deficiency, iodine deficiency further aggravates the condition and carries a great potential to show deleterious impacts on maternal and fetal outcomes, thus serving a major health problem. Considering the substantial increase in short term as well as long term perinatal morbidity, a felt need for creating awareness not only on adverse outcomes but also detection of the disorder in first trimester is required. The desired perinatal outcome can be achieved by early detection and prompt treatment of the disorder. Henceforth, universal screening of all antenatal women in the first trimester rather than targeted case finding should be recommended at all the health care facilities. Moreover, this message should be clearly percolated at all tiers of health care system.

FIGO working group on good clinical practice in maternal-fetal medicine 2015-2018 states that pregnancy acts a stress test for thyroid gland and results in hypothyroidism in women who are iodine deficient or have inadequate thyroid reserve, and postpartum thyroiditis in previously euthyroid women with underlying hashimoto's thyroiditis.

Funding: No funding sources Conflict of interest: None declared

Ethical approval: The study was approved by the Institutional Ethics Committee

\section{REFERENCES}

1. Gupta K. Thyroid disorders and pregnancy. FOGSI FOCUS-Med Dis Preg. 2009;10:59-66.

2. Sreelatha S, Nadagoudar S, Devi L, Asha. The study of maternal and fetal outcome in pregnant women with thyroid disorders. Int J Reprod Contracept Obstet Gynecol. 2017;6:3507.

3. Dhanwal DK, Bajaj S, Rajput R, Subramaniam KA, Chowdhury S, Bhandari R, et al. Prevalence of hypothyroidism in pregnancy: An epidemiological study from 11 cities in 9 states of India. Indian $\mathbf{J}$ Endocrinol Metabol. 2016;20(3):387.

4. Vaidya B, Anthony S, Bilous M, Shields B, Drury J, Hutchiso S, et al. Detection of thyroid dysfunction in early pregnancy Universal screening or targeted high risk case finding? J Clin Endocrinol Metab. 2007;92-203-7.

5. Negro R, Schwartz A, Gismondi R, Tinelli A, Mangieri T, Stagnaro-Green A. Universal screening vs case finding for detection and treatment of thyroid hormonal dysfunction 
during pregnancy. J Clin Endocrinol Metab. 2010;95:1699707.

6. Meena DS, Bhati I, Bora S, Meena S. Study of thyroid dysfunction in pregnancy. Int J Curr Microbiol App Sci. 2015;4(9):91-7.

7. Nazarpour S, Ramezani Tehrani F, Simbar M, Azizi F. Thyroid dysfunction and pregnancy outcomes. Iran J Reprod Med. 2015;13(7):387-96.

8. FOGSI good clinical practice recommendations on pre conceptional care- India; 2016;4(2.1):6-7.

9. Indian thyroid society, FOGSI. Recommendations for the management of thyroid dysfunction in pregnancy. 2019:11-12.

10. Lazarus J, Brown RS, Daumerie C, HubalewskaDydejczyk A, Negro R, Vaidya B. 2014 European thyroid association guidelines for the management of subclinical hypothyroidism in pregnancy and in children. Eur Thyroid J. 2014;3(2):76-94.

11. Sastre-Marcos J, Val-Zaballos F, Ruiz-Ginés MÁ, SauraMontalbán J, Veganzones-Pérez M. Reference values and universal screening of thyroid function in the first trimester of the population of pregnant women in Toledo (Spain). Endocrinologia nutricion (English Edition). 2015;7(62):358-60.

12. NHM. National Guidelines for Screening of hypothyroidism during pregnancy, Ministry of Health and Family Welfare, Government of India; 2014.

13. Stagnaro-Green A, Abalovich M, Alexander E, Azizi F, Mestman J, Negro R, et al. Guidelines of the American thyroid association for the diagnosis and management of thyroid disease during pregnancy and postpartum. Thyroid. 2011;21(10):1081-125.
14. Lazarus JH, Bestwick JP, Channon S, Paradice R, Maina A, Rees R, et al. Antenatal thyroid screening and childhood cognitive function. $N$ Eng $J$ Med. 2012;366(6):493-501.

15. Tanuja PM, Rajgopal K, Sadiqunnisa. Thyroid dysfunction in pregnancy and maternal outcome. IOSR-JDMS. 2014;13(1):11-5.

16. Kalra B, Choudhary M, Thakral M, Kalra S. Prevalence of hypothyroidism in term pregnancies in North India. Indian J Endocr Mtab. 2018;22:13-5.

17. Ozdemir H, Akman I, Coskun S, Demirel U, Turan S, Bereket A, et al. Maternal thyroid dysfunction and Neonatal thyroid problems. Int $J$ Endocrinol. 2013;2013:987843.

18. Rajalakshmi MS, Singh AK, Sujit D, Samchetsabam RD. The fetomaternal outcome in maternal hypothyroidism complicating pregnancies at RIMS: a prospective study. Eur J Pharm Med Res. 2016;3:452-9.

19. Gupta RD. Thyroid disorders in pregnancy. Curr Med Issues. 2018;16:48-51.

20. Saki F, Dabbaghmanesh MH, Ghaemi SZ, Forouhari S, Omrani GR, Bakhshayeshkaram M. Thyroid function in pregnancy and its influences on maternal and fetal outcomes. Int J Endocrinol Metab. 2014;12(4):e19378.

Cite this article as: Agarwal J, Kesaraju D, Badkur P. Maternal outcome in thyroid dysfunction in admitted antenatal women in tertiary care centre: an observational study. Int J Reprod Contracept Obstet Gynecol 2020;9:3402-7. 